\title{
Thermal Expansion of Carbamazepine: Systematic Crystallographic Measurements Challenge Quantum Chemical Calculations
}

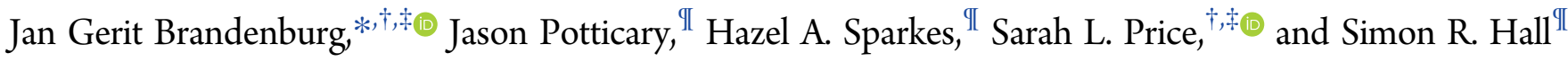 \\ ${ }^{\dagger}$ Department of Chemistry, University College London, 20 Gordon Street, London WC1H 0AH, United Kingdom \\ IThomas Young Centre, University College London, Gower Street, London WC1E 6BT, United Kingdom \\ ${ }^{\star}$ School of Chemistry, University of Bristol, Cantocks Close, Bristol BS8 1TS, United Kingdom
}

Supporting Information

\begin{abstract}
We report systematic temperature-dependent X-ray measurements on the most stable carbamazepine polymorph. This active pharmaceutical ingredient is used to demonstrate how the thermal expansion can probe certain intermolecular interactions resulting in anisotropic expansion behavior. We show that most structural features can be captured by electronic structure calculations at the quasi-harmonic approximation (QHA) provided a dispersion-corrected density functional based method is employed. The impact of thermal expansion on the phonon modes and hence free energy contributions is large enough to impact the relative stability of different polymorphs.
\end{abstract}

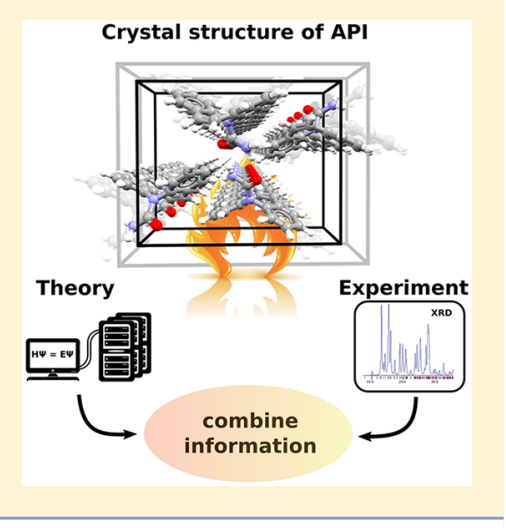

$\mathrm{T}$ he pharmaceutical industry is increasingly looking toward computational methods to assist in drug development. The aim is to shorten the time needed for establishing the physical properties of the crystalline forms that are relevant to designing the formulation and manufacturing process. The thermal expansion properties are very specific to the polymorph as well as the molecule, and the difference in the anisotropy can be significant. The thermal expansion of the chosen solid form of the active pharmaceutical ingredient (API) can affect whether the formulated pills maintain their integrity over the wide range of temperatures involved in manufacture, storage, and transport throughout the world. ${ }^{1,2}$ However, thermal expansion may also play a significant role in the computation of more fundamental properties. The crystal structure obtained by lattice energy optimization differs from the experimental structure by the thermal expansion, and this variation can have a pronounced effect on the corresponding powder diffraction pattern causing problems in using computed structures to help identify novel solid forms. ${ }^{3,4}$ The relative stability is a vital property, and the inclusion of thermal expansion and zero-point effects has been estimated to change the relative stability of $21 \%$ of 475 known pairs of polymorphs. ${ }^{5}$ We have recently shown that the neglect of thermal expansion can lead to a significant underestimation of the calculated heat capacities of naproxen, which differ sufficiently to affect the relative stability of the racemic and enantiopure forms. ${ }^{6}$ Hence, it is important to measure the thermal expansion of pharmaceutical crystals to assess what approximations in computational treatment of thermal expansion are appropriate for various applications.
Density functional theory (DFT) has widespread use in computational materials science and cognate disciplines. ${ }^{7}$ The development of London dispersion corrections in the DFT framework extended their applicability to the important class of noncovalently interacting systems like organic crystals. ${ }^{8-10}$ Dispersion corrected DFT (DFT-D) has been tested extensively on the computation of electronic lattice energies with mean absolute errors of 4-10 kJ/mol. ${ }^{11-14}$ For modeling the temperature-dependent thermodynamic properties, the quasi-harmonic approximation (QHA) has been shown to be more efficient than molecular dynamics and more realistic than the harmonic approximation as it includes thermal expansion. ${ }^{15}$ In recent years, the QHA has been applied using classical force fields, ${ }^{16,17}$ periodic electronic structure calculations both by DFT-D methods, ${ }^{14,18-20}$ and post-Hartree-Fock methods. ${ }^{21,22}$ While the post-Hartree-Fock studies focused on small molecular crystals, a recent study on purine has pioneered the thermal expansion on an organic solid, namely, purine. ${ }^{20}$ The present paper also generates the experimental data needed to assess the QHA for a more typical pharmaceutical crystal.

Realistic pharmaceutical crystals are significantly larger, and their modeling requires more efficient simulation methods. Thus, recent efforts provided the chemistry community with new electronic structure methods, which cover several orders of magnitude in computational speed. ${ }^{23-25}$ While good structures and energies for organic crystals have been presented, careful

Received: July 26, 2017

Accepted: August 25, 2017

Published: August 25, 2017 
(a)

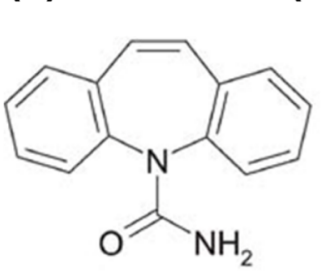

(b)

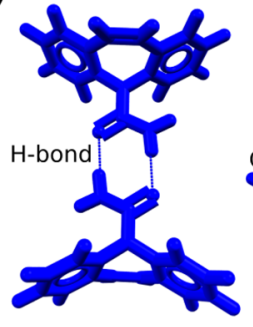

(c)

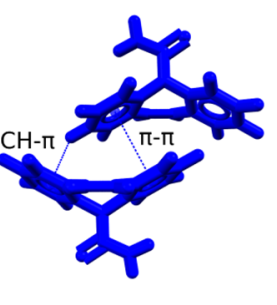

(d)

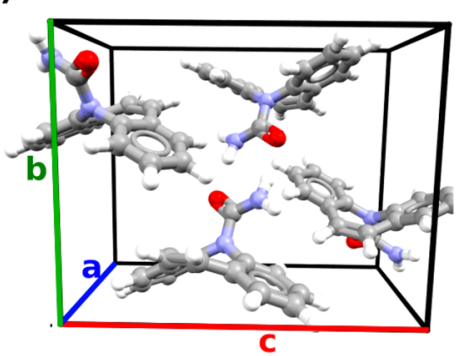

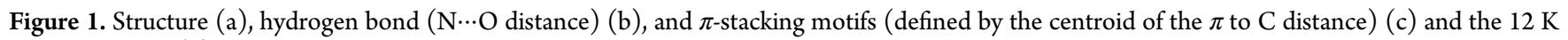
X-ray structure (d) of carbamazepine form III, showing the crystallographic directions.

tests are needed to establish their broad applicability to second-

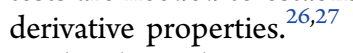

The thermal expansion of pharmaceutical crystals reflects a complex balance between various intermolecular forces, such as hydrogen and halogen bonding, $\pi \cdots \pi$ stacking, van der Waals forces, and changes in the conformation and covalent bonding. The example chosen was carbamazepine (Figure 1), a small antiepileptic, antioconvulsant, bipolar disorder treatment drug that has both polar, hydrogen bonding groups and a large aromatic fragment. Four carbamazepine polymorphs contain the same hydrogen-bonded dimer but vary in the aromatic packing of the hydrocarbon dibenzoazepine rings. In contrast, form V has catermeric hydrogen bonding. ${ }^{28}$ Over 50 crystalline structures containing carbamazepine have been produced. ${ }^{29} \mathrm{We}$ chose to study the crystal structure of form $\mathrm{III}^{30}$ as it is the most stable at ambient temperatures, though the energy separation of the dimeric polymorphs (I-IV) is less than 3 $\mathrm{kJ} / \mathrm{mol}^{31}$ There have been many previous determinations of the form III structure at room temperature, but the lowesttemperature study was to determine the electron density at 100 $\mathrm{K}^{32,33}$ We measure the variation in the structure of carbamazepine form III between 12 and $298 \mathrm{~K}$ from powder $\mathrm{X}$-ray data and use this thermal expansion data to assess whether the affordable electronic structure methods within the QHA can predict the effect of molecular motions on the crystal structure.

Carbamazepine was purchased from Sigma-Aldrich (C4024) and purified via recrystallization using ethanol/water as a solvent/antisolvent mixture. The standard form III polymorph was obtained by slow cooling $\left(60{ }^{\circ} \mathrm{C}\right.$-ambient over $\left.24 \mathrm{~h}\right)$ of a saturated solution of purified carbamazepine in ethanol, which gave small white rhombohedral crystals. The form III crystals were gently ground into a fine powder before being mounted on a chromium-plated copper sample holder and loaded into an Oxford Instruments low-temperature PheniX stage. A Bruker D8 Advance diffractometer with a PSD LynxEye detector and Oxford Cryosystems pHeniX cryostat was used. X-ray diffraction of the powder was measured over the angle range of $5-35^{\circ} 2 \theta$ with a step size of $0.02^{\circ}$ and collection time of 20 min continuously over 4 days as the temperature was lowered from 298 to $12 \mathrm{~K}$. Unit cell parameters were calculated by Rietveld refinement of the known form III powder pattern with the software Profex using the BGMN engine. ${ }^{34}$ Refinement was considered complete when $R_{\mathrm{wp}} \leq 1.5 R_{\text {exp }}$.

The QHA introduces the missing volume dependence of phonon frequencies by retaining the harmonic expression for the Helmholtz free energy

$$
F^{\mathrm{QHA}}(T, V)=E_{\mathrm{latt}}(V)+F_{\mathrm{vib}}^{\mathrm{QHA}}(T, V)
$$

Here, $E_{\text {latt }}(V)$ is the zero-temperature internal energy of the crystal (its lattice energy) without any vibrational contribution with the cell and atomic positions optimized at fixed volume $V$. The vibrational contributions are

$$
\begin{aligned}
F_{\text {vib }}^{\mathrm{QHA}}(T, V)= & \sum_{\mathbf{k}, p} \frac{\hbar \omega_{\mathbf{k}, p}(V)}{2} \\
& +k_{\mathrm{B}} T \sum_{\mathbf{k}, p}\left[\ln \left(1-\mathrm{e}^{-\hbar \omega_{\mathbf{k}, p}(V) / k_{\mathrm{B}} T}\right)\right]
\end{aligned}
$$

where the phonon frequencies $\omega_{\mathbf{k}, p}$, which correspond to a $\mathbf{k}$ point in the first Brillouin zone and a phonon band index $p$, depend on the unit cell volume. Table S9 tests the effect of sampling phonons within the first Brilloiun zone. The equilibrium volume at a given temperature $T, V(T)$, is obtained by minimizing $F^{\mathrm{QHA}}(T, V)$ with respect to the volume $V$ at fixed $T$. This optimization varies the cell parameters for each fixed volume and therefore gives expansion of the crystallographic directions based on a static lattice $\left(E_{\text {latt }}\right)$ not a dynamic lattice $(F)$. Because it is desirable to find an optimal compromise between accuracy and CPU time, we apply the following three methods, with decreasing computational cost:

(1) Screened exchange hybrid functional in a medium-sized basis set with corrections for London dispersion and basis set errors (HSE-3c) 25,35

(2) Minimal basis set Hartree-Fock with corrections for London dispersion and basis set errors $(\mathrm{HF}-3 \mathrm{c})^{24,36}$

(3) Simplified density functional tight-binding method of third order with self-consistent charges and London dispersion correction (DFTB3-D3) $37-39$

For our system, the fastest method, DFTB3-D3, is a factor of 2700 faster than the most expensive, HSE-3c. DFTB3-D3 is a standard semiempirical approach with quite severe approximations in the many center integrals. ${ }^{40}$ In contrast, HSE-3c has been shown to be competitive with standard dispersioncorrected density functional approximations in converged basis set expansions ${ }^{25,35}$ and superior to other approaches employing small basis sets. ${ }^{35,41}$ Tables S1 and S7 demonstrate that accuracy also applies to the carbamazepine crystal. The methods are implemented in a developer version of the CRYSTAL $^{42,43}$ program suite, which scales well on highperformance computing facilities. ${ }^{44}$ However, for this study, CPU time of about $10000 \mathrm{~h}$ was sufficient to perform the calculations (explicit timing in Supporting Information). For the QHA, a convenient module can be used that automatically performs the required $V$-constrained optimizations, phonon computations, interpolation of modes, and subsequent computation of desired properties. ${ }^{45-48}$ 
Table 1. Freely Relaxed and Zero-Point Inclusive Computed Crystal Structures Compared to Low-Temperature X-ray Structure $(12 \mathrm{~K})^{a}$

\begin{tabular}{|c|c|c|c|c|c|c|}
\hline & $a / \AA$ & $b / \AA$ & $c / \AA$ & $\beta / \operatorname{deg}$ & $\mathrm{vol} / \AA^{3}$ & $\mathrm{RMSD} 15 / \AA$ \\
\hline $\mathrm{X}$-ray $[12 \mathrm{~K}]$ & 7.51 & 11.07 & 13.79 & 92.9 & 1145.2 & \\
\hline \multicolumn{7}{|c|}{0 K, Zero-Point Exclusive (Lattice Energy) } \\
\hline DFTB3-D3 & 7.54 & 10.23 & 12.95 & 92.9 & 997.7 & 0.53 \\
\hline $\mathrm{HF}-3 \mathrm{c}$ & 7.44 & 10.54 & 13.29 & 92.9 & 1040.1 & 0.37 \\
\hline HSE-3c & 7.49 & 10.95 & 13.57 & 93.1 & 1111.4 & 0.14 \\
\hline PBE-D3/SVP & 7.47 & 10.78 & 13.44 & 92.8 & 1081.4 & 0.22 \\
\hline \multicolumn{7}{|c|}{$0 \mathrm{~K}$, Zero-Point Inclusive } \\
\hline DFTB3-D3 & 7.61 & 10.29 & 13.03 & 92.9 & 1017.6 & 0.51 \\
\hline $\mathrm{HF}-3 \mathrm{c}$ & 7.48 & 10.60 & 13.40 & 92.9 & 1064.2 & 0.34 \\
\hline HSE- $3 c$ & 7.54 & 11.02 & 13.68 & 93.1 & 1137.2 & 0.09 \\
\hline PBE-D3/SVP & 7.52 & 10.84 & 13.55 & 92.8 & 1104.9 & 0.17 \\
\hline
\end{tabular}

The cell volume and cell parameters have been measured between 12 and $298 \mathrm{~K}$ in about $1.5 \mathrm{~K}$ steps; refined crystallographic information files are provided in the Supporting Information. A comparison of the low-temperature (12K) and room-temperature $(298 \mathrm{~K})$ structures shows that the carbamazepine molecular conformation does not change upon heating. However, the carbamazepine molecule is enlarged, with the rotational constants decreasing by about $2.3 \%$. This is not reproduced by our calculations (see Figure S1 and Table S6). Perturbative anharmonic treatment using DFT is able to compute the expansion of small organic molecules in the gas phase. ${ }^{49}$ Hence, it is not clear how much of the error comes from averaging over the modes in the diffraction experiment, the use of the QHA, or the electronic structure theory used (see Table S7). Analyzing the intermolecular interactions, we see several different features that expand in a similar fashion and are well represented by our computational approach. The hydrogen bond distance, the $\pi-\pi$ stacking of the parallel aromatic rings, and the $\mathrm{CH}-\pi$ distance of the approximately orthogonal aromatic parts (see Figure 1) expand by 1.0, 1.3, and $1.2 \%$, respectively, between 12 and $300 \mathrm{~K}$ (see Table S8). We observe that the surprisingly similar expansion of the $\mathrm{H}$ bond and the $\pi$-stacking motifs can be attributed to the interlocking of carbamazepine molecules within this particular crystal, preventing independent changes in these packing motifs.

Additional qualitative insight can be gained by comparing the Grüneisen parameter $\gamma_{i}=-\left(V / \omega_{i}\right)\left(\partial \omega_{i} / \partial V\right)$, which quantifies change in the phonon modes, as computed by HSE-3c and given in Table S10. The stiffening of the $\mathrm{N}-\mathrm{H}$ stretching modes $(\gamma<0)$ indicates a weakening of the hydrogen bond with increasing temperature. In contrast, the modes corresponding to a relative movement of the $\pi$-stacked molecules have the highest Grüneisen parameter $(\gamma>0)$ and are softening. This softening of low-frequency modes contributes significantly to the entropy gain upon heating up the crystal. Careful identification of the specific modes was required to calculate the Grüneisen parameter as several modes cross in energy (see Figure S5). There is no gap between inter- and intramolecular modes, in contrast to small rigid molecular crystals like benzene. This indicates that the phonon spectrumdependent free energy contributions are more challenging to describe, and it seems unlikely that a separation of modes, as in a rigid-body molecule approximation, will be accurate for pharmaceutical-like organic crystals. ${ }^{6}$
A quantitative comparison of the cell parameters at $12 \mathrm{~K}$ and the lattice energy minimum (Table 1) shows a systematic improvement from DFTB3-D3 to HF-3c to HSE-3c, which is most evident from the comparison of a 15 molecule coordination cluster (RMSD15). Indeed, the HSE-3c structure is slightly better than the geometry from PBE-D $3^{51,52}$ evaluated in a medium-sized def2-SVP basis set. ${ }^{53}$ The computed unit cells from lattice energy optimizations are smaller than the $\mathrm{X}$ ray structure. This is correct as the zero-point vibrational contribution enlarges the computed cells even at $0 \mathrm{~K}$ (Table 1 ). The volume expansion due to zero-point vibrations is a significant $2 \%$ for all methods. This agrees well with previous studies on crystals of smaller molecules, where the zero-point expansion ranges between 2.1 and $3.3 \%{ }^{19,22,54}$ and the dependence of the errors on the specific cell length further emphasizes the problems of assessing computational methods on the basis of computed volumes. ${ }^{55}$

In Figure 2, we show the thermal expansion of the carbamazepine crystal from the three electronic structure methods compared to our measurements. The screened exchange hybrid functional HSE-3c performs well, but DFTB3-D3 is poor in absolute terms. However, the relative thermal volume expansion is captured well by all methods (Figure S3), varying from 3.0 and 3.6\% from DFTB3-D3 and HSE-3c, respectively, both agreeing well with the experimental $3.6 \%$ between 12 and $298 \mathrm{~K}$.

A more detailed analysis of the thermal expansion of the individual unit cell parameters is shown in Figure 2 for the absolute cell parameters for the HSE-3c method. On the absolute scale, one can mainly see that the cell parameter computed by HSE-3c agrees well with the measurements; the deviations are well below $1 \%$ for all directions. The relative deviations (Figure S4) show some more pronounced differences. While the $c$ direction is correctly computed to expand the most, the calculations do not differentiate between the other two crystallographic directions, which expand by $0.8 \%$ in the $a$ direction and $1.3 \%$ in the $b$ direction. This might indicate that the theoretical approach is too approximate to capture all of the interactions within the crystal to the same accuracy. In particular, the different stacking motifs sketched in Figure 1 have to be described in a balanced way to capture the overall intermolecular interactions, let alone how the conformation and vibrations of the molecule are affected by the crystal packing.

Overall, we have good agreement of the computed and measured structural features computed by our most accurate HSE-3c electronic structure method, while the cheapest 

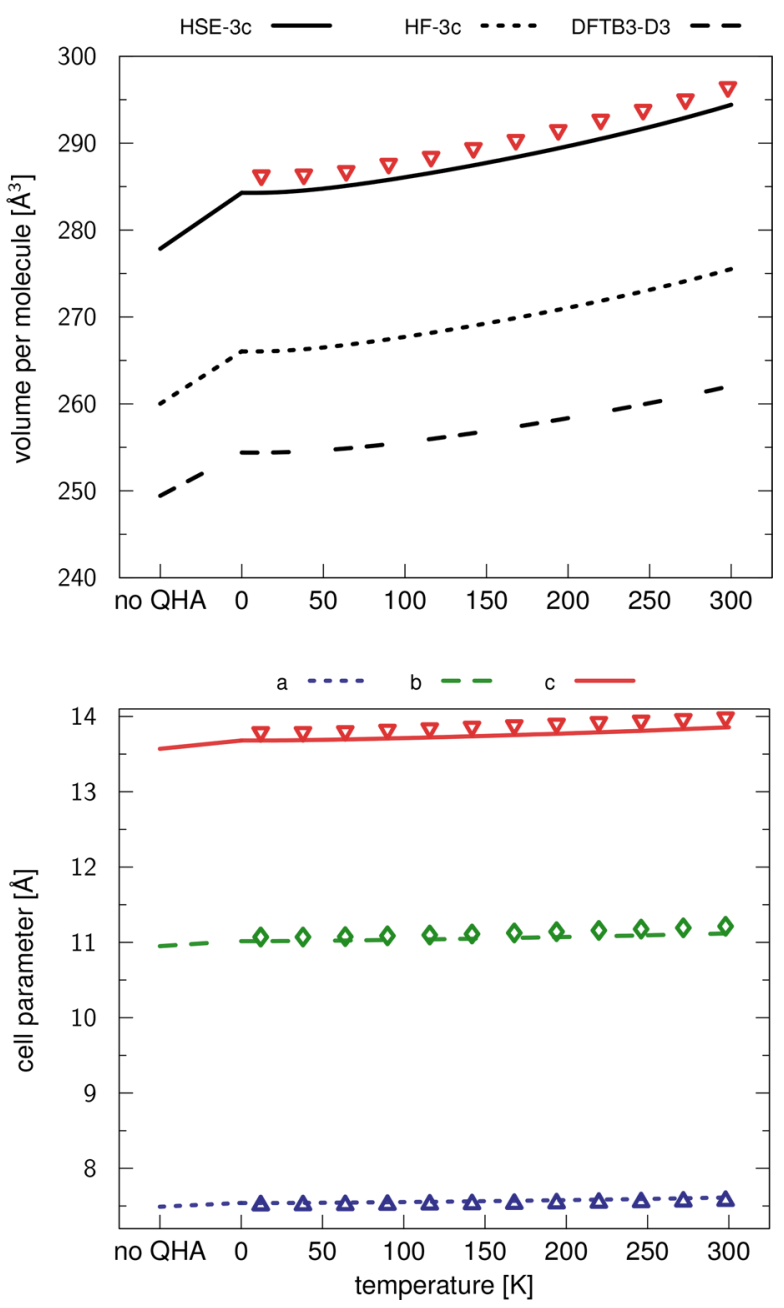

Figure 2. Temperature dependency of computed unit cell volumes with different electronic structure methods (top) and of the HSE-3ccomputed cell parameters (bottom) in comparison to the measured $\mathrm{X}$ ray structures (open symbols). The difference between no QHA and 0 $\mathrm{K}$ is the zero-point expansion, as quantified in Table 1.

DFTB3-D3 scheme proved to be insufficiently accurate. For relative unit cell expansions, all three methods give reasonably good agreement, while none gave the difference in the $a$ and $b$ crystallographic directions accurately. Other inaccuracies in the computational approach are due the QHA and possibly the restricted phonon mode sampling. The mixing of the inter- and intramolecular modes shows that assuming that all of the anhamonicity in all modes is small is questionable. Indeed, for other organic crystals, the thermal ellipsoids may show that some modes are very poorly described by the QHA. Depending on the specific crystal structure, quantitative accurate calculation of thermal expansion might need more elaborate methods, for example, explicit treatment of the different directions, that is, one would need to construct the free energy potential $F_{\mathrm{QHA}}$ as a function of the unit cell parameters. Some crystals may even require sampling of the full potential energy surface via molecular dynamics simulations. ${ }^{54}$

While the prediction of correct thermal expansion is important, it is even more important to assess the effect on the relative free energies of polymorphs. In Figure 3, we show the change of the sublimation enthalpy and free energy with temperature. At higher temperatures, the entropy contributions dominate, but the zero-point energy is still significant. The
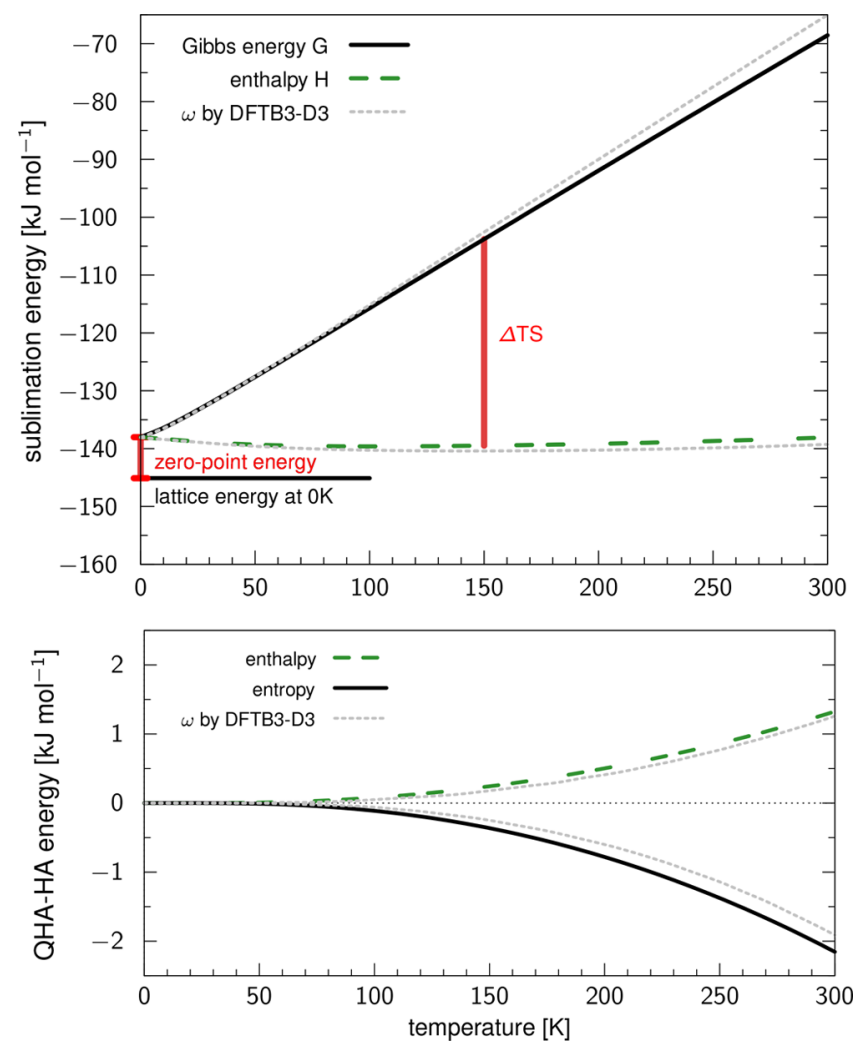

Figure 3. Top: Computed Gibbs free energy $G$ and enthalpy $H$ as a function of temperature at the HSE-3c level within the QHA. The contributions of the lattice energy, zero-point energy, and entropy are highlighted. Bottom: The difference in enthalpy and entropy (TS) contributions from the QHA vs HA approach is shown. Results by combining the HSE-3c lattice energy with zero-point and free energy contributions from DFTB3-D3 are shown as gray dotted lines.

lower panel of Figure 3 shows the differences in enthalpy and entropy when computed in the QHA, which includes thermal expansion, and in the $\mathrm{HA}$, which ignores it. At room temperatures, we note differences of $1-2 \mathrm{~kJ} / \mathrm{mol}$ in the enthalpic and entropic contributions that partially cancel each other for carbamazepine form III. These contributions are within the typical range of polymorphic energy differences ${ }^{2}$ and thus important to consider, particularly when the two polymorphs have pronounced differences in their conformations and packing motifs and thus the two crystals could have significant differences in the anisotropy of their thermal expansion. Figure 3 also shows zero-point, enthalpy, and entropy contributions based on DFTB3-D3 phonon calculations combined with the lattice energy from HSE-3c. As thousands of individual frequencies have to be calculated, this is the computational bottleneck and using DFTB3-D3 speeds up the calculations significantly. The results are in close agreement with the full HSE-3c treatment and therefore sufficient for estimating free energy and relative thermal expansion for many purposes.

We have presented consistent temperature-dependent crystallographic measurements of carbamazepine form III. These rare thermal expansion data highlight the anisotropic inter- and intramolecular interactions in this prototypical API. We find that different interaction motifs like H-bonding and $\pi-\pi$ stacking are strongly coupled, leading to similar thermal expansion of all intermolecular distances for this specific polymorph. Some subtle differences in the anisotropy of the 
thermal expansion are not captured by the QHA using low-cost electronic structure methods. However, calculating the phonons by DFTB3-D3 presents an efficient route for estimating the contributions to the free energy. However, we have only studied one particular polymorph of a nearly rigid molecule in which the packing couples the different intermolecular interactions. Hence, determining whether this approach can provide reliable free energy differences for pharmaceutical polymorphs requires similar joint experimental and theoretical studies for a wide range of organic systems. Hence, determining whether this approach can provide reliable free energy differences for polymorphs of small pharmaceutical molecules in industrial development requires similar joint experimental and theoretical studies for a wide range of organic systems.

\section{ASSOCIATED CONTENT}

\section{S Supporting Information}

The Supporting Information is available free of charge on the ACS Publications website at DOI: 10.1021/acs.jpclett.7b01944.

Details of the computational setup, experimental data, and additional theoretical results, including a list of the Grüneisen parameters (PDF)

Crystallographic information file on measured X-ray structures at $12,50,100,200$, and $298 \mathrm{~K}$ (CIF)

\section{AUTHOR INFORMATION}

\section{Corresponding Author}

*E-mail: g.brandenburg@ucl.ac.uk.

\section{ORCID}

Jan Gerit Brandenburg: 0000-0002-9219-2948

Sarah L. Price: 0000-0002-1230-7427

\section{Notes}

The authors declare no competing financial interest.

\section{ACKNOWLEDGMENTS}

Our work is supported by MagnaPharm, a collaborative research project funded by the European Union's Horizon 2020 Research and Innovation programme under Grant Agreement Number 736899. S.R.H. and J.P. acknowledge the Engineering and Physical Sciences Research Council (EPSRC), U.K. (Grant EP/G036780/1), and the Bristol Centre for Functional Nanomaterials for support. J.G.B acknowledges support by the Alexander von Humboldt foundation within the Feodor-Lynen program.

\section{REFERENCES}

(1) Elder, D. P.; Patterson, J. E.; Holm, R. The solid-state continuum: a perspective on the interrelationships between different solid-state forms in drug substance and drug product. J. Pharm. Pharmacol. 2015, $67,757-772$.

(2) Cruz-Cabeza, A. J.; Reutzel-Edens, S. M.; Bernstein, J. Facts and fictions about polymorphism. Chem. Soc. Rev. 2015, 44, 8619-8635.

(3) Habermehl, S.; Mörschel, P.; Eisenbrandt, P.; Hammer, S. M.; Schmidt, M. U. Structure determination from powder data without prior indexing, using a similarity measure based on cross-correlation functions. Acta Crystallogr., Sect. B: Struct. Sci., Cryst. Eng. Mater. 2014, $70,347-359$.

(4) Stephenson, G. A. Anisotropic Lattice Contraction in Pharmaceuticals: The Influence of Cryo-Crystallography on Calculated Powder Diffraction Patterns. J. Pharm. Sci. 2006, 95, 821-827.
(5) Nyman, J.; Day, G. M. Modelling temperature-dependent properties of polymorphic organic molecular crystals. Phys. Chem. Chem. Phys. 2016, 18, 31132-31143.

(6) Buchholz, H.; Hylton, R. K.; Brandenburg, J. G.; SeidelMorgenstern, A.; Lorenz, H.; Stein, M.; Price, S. L. The thermochemistry of racemic and enantiopure molecular crystals for predicting enantiomer separation. Cryst. Growth Des. 2017, DOI: $10.1021 /$ acs.cgd.7b00582.

(7) Curtarolo, S.; Hart, G. L. W.; Nardelli, M. B.; Mingo, N.; Sanvito, S.; Levy, O. The high-throughput highway to computational materials design. Nat. Mater. 2013, 12, 191-201.

(8) Klimeš, J.; Michaelides, A. Perspective: Advances and challenges in treating van der Waals dispersion forces in density functional theory. J. Chem. Phys. 2012, 137, 120901.

(9) Grimme, S.; Hansen, A.; Brandenburg, J. G.; Bannwarth, C. Dispersion-Corrected Mean-Field Electronic Structure Methods. Chem. Rev. 2016, 116, 5105-5154.

(10) Hoja, J.; Reilly, A. M.; Tkatchenko, A. First-principles modeling of molecular crystals: structures and stabilities, temperature and pressure. WIREs Comput. Mol. Sci. 2017, 7, e1294.

(11) Otero-de-la-Roza, A.; Johnson, E. R. A Benchmark for NonCovalent Interactions in Solids. J. Chem. Phys. 2012, 137, 054103.

(12) Reilly, A. M.; Tkatchenko, A. Understanding the Role of Vibrations, Exact Exchange, and Many-Body van der Waals Interactions in the Cohesive Properties of Molecular Crystals. J. Chem. Phys. 2013, 139, 024705.

(13) Brandenburg, J. G.; Grimme, S. Organic crystal polymorphism: A benchmark for dispersion corrected mean field electronic structure methods. Acta Crystallogr., Sect. B: Struct. Sci., Cryst. Eng. Mater. 2016, $72,502-513$.

(14) Brandenburg, J. G.; Maas, T.; Grimme, S. Benchmarking DFT and Semiempirical Methods on Structures and Lattice Energies for ten Ice Polymorphs. J. Chem. Phys. 2015, 142, 124104.

(15) Dove, M. T. Introduction to Lattice Dynamics; Cambridge University Press: Cambridge, U.K., 1993.

(16) Dybeck, E. C.; Abraham, N. S.; Schieber, N. P.; Shirts, M. R. Capturing Entropic Contributions to Temperature-Mediated Polymorphic Transformations Through Molecular Modeling. Cryst. Growth Des. 2017, 17, 1775-1787.

(17) Nyman, J.; Pundyke, O. S.; Day, G. M. Accurate force fields and methods for modelling organic molecular crystals at finite temperatures. Phys. Chem. Chem. Phys. 2016, 18, 15828.

(18) Červinka, C.; Fulem, M.; Stoffel, R. P.; Dronskowski, R. Thermodynamic Properties of Molecular Crystals Calculated within the Quasi-Harmonic Approximation. J. Phys. Chem. A 2016, 120, 2022-2034.

(19) Erba, A.; Maul, J.; Civalleri, B. Thermal properties of molecular crystals through dispersion-corrected quasi-harmonic ab initio calculations: the case of urea. Chem. Commun. 2016, 52, 1820-1823.

(20) Ruggiero, M. T.; Zeitler, J. A.; Erba, A. Intermolecular anharmonicity in molecular crystals: interplay between experimental low-frequency dynamics and quantum quasi-harmonic simulations of solid purine. Chem. Commun. 2017, 53, 3781-3784.

(21) Heit, Y. N.; Nanda, K. D.; Beran, G. J. O. Predicting finitetemperature properties of crystalline carbon dioxide from first principles with quantitative accuracy. Chem. Sci. 2016, 7, 246-255.

(22) Heit, Y. N.; Beran, G. J. O. How important is thermal expansion for predicting molecular crystal structures and thermochemistry at finite temperatures? Acta Crystallogr., Sect. B: Struct. Sci., Cryst. Eng. Mater. 2016, 72, 514-529.

(23) Brandenburg, J. G.; Grimme, S. Accurate Modeling of Organic Molecular Crystals by Dispersion-Corrected Density Functional Tight Binding (DFTB). J. Phys. Chem. Lett. 2014, 5, 1785-1789.

(24) Sure, R.; Grimme, S. Corrected Small Basis Set Hartree-Fock Method for Large Systems. J. Comput. Chem. 2013, 34, 1672-1685.

(25) Brandenburg, J. G.; Caldeweyher, E.; Grimme, S. Screened exchange hybrid density functional for accurate and efficient structures and interaction energies. Phys. Chem. Chem. Phys. 2016, 18, 1551915523. 
(26) Schaub, T. A.; Sure, R.; Hampel, F.; Grimme, S.; Kivala, M. Quantum Chemical Dissection of the Shortest P $=\mathrm{O} \cdots \mathrm{I}$ Halogen Bond: The Decisive Role of Crystal Packing Effects. Chem. - Eur. J. 2017, 23, 5687-5691.

(27) Rösel, S.; Quanz, H.; Logemann, C.; Becker, J.; Mossou, E.; Cañadillas Delgado, L.; Caldeweyher, E.; Grimme, S.; Schreiner, P. R. London Dispersion Enables the Shortest Intermolecular Hydrocarbon H $\cdots$ H Contact. J. Am. Chem. Soc. 2017, 139, 7428-7431.

(28) Arlin, J.-B.; Price, L. S.; Price, S. L.; Florence, A. J. A strategy for producing predicted polymorphs: catemeric carbamazepine form $\mathrm{V}$. Chem. Commun. 2011, 47, 7074-7076.

(29) Childs, S. L.; Wood, P. A.; Rodriguez-Hornedo, N.; Reddy, L. S.; Hardcastle, K. I. Analysis of 50 Crystal Structures Containing Carbamazepine Using the Materials Module of Mercury CSD. Cryst. Growth Des. 2009, 9, 1869-1888.

(30) Himes, V. L.; Mighell, A. D.; De Camp, W. H. Structure of carbamazepine: $5 H$-dibenz $[b, f]$ azepine-5-carboxamide. Acta Crystallogr., Sect. B: Struct. Crystallogr. Cryst. Chem. 1981, 37, 2242-2245.

(31) Grzesiak, A. L.; Lang, M.; Kim, K.; Matzger, A. J. Comparison of the four anhydrous polymorphs of carbamazepine and the crystal structure of form I. J. Pharm. Sci. 2003, 92, 2260-2271.

(32) El Hassan, N.; Ikni, A.; Gillet, J.-M.; Spasojevic-de Bire, A.; Ghermani, N. E. Electron Properties of Carbamazepine Drug in Form III. Cryst. Growth Des. 2013, 13, 2887-2896.

(33) Sovago, I.; Gutmann, M. J.; Senn, H. M.; Thomas, L. H.; Wilson, C. C.; Farrugia, L. J. Electron density, disorder and polymorphism: high-resolution diffraction studies of the highly polymorphic neuralgic drug carbamazepine. Acta Crystallogr., Sect. B: Struct. Sci., Cryst. Eng. Mater. 2016, 72, 39-50.

(34) Döbelin, N.; Kleeberg, R. Profex: a graphical user interface for the Rietveld refinement program BGMN. J. Appl. Crystallogr. 2015, 48, $1573-1580$.

(35) Grimme, S.; Brandenburg, J. G.; Bannwarth, C.; Hansen, A. Consistent structures and interactions by density functional theory with small atomic orbital basis sets. J. Chem. Phys. 2015, 143, 054107.

(36) Cutini, M.; Civalleri, B.; Corno, M.; Orlando, R.; Brandenburg, J. G.; Maschio, L.; Ugliengo, P. Assessment of Different Quantum Mechanical Methods for the Prediction of Structure and Cohesive Energy of Molecular Crystals. J. Chem. Theory Comput. 2016, 12, $3340-3352$

(37) Elstner, M.; Porezag, D.; Jungnickel, G.; Elsner, J.; Haugk, M.; Frauenheim, T.; Suhai, S.; Seifert, G. Self-Consistent-Charge DensityFunctional Tight-Binding Method for Simulations of Complex Materials Properties. Phys. Rev. B: Condens. Matter Mater. Phys. 1998, 58, 7260-7268.

(38) Aradi, B.; Hourahine, B.; Frauenheim, T. DFTB+, a Sparse Matrix-Based Implementation of the DFTB Method. J. Phys. Chem. A 2007, 111, 5678-5684.

(39) Gaus, M.; Goez, A.; Elstner, M. Parametrization and Benchmark of DFTB3 for Organic Molecules. J. Chem. Theory Comput. 2013, 9, $338-354$.

(40) Christensen, A. S.; Kubař, T.; Cui, Q.; Elstner, M. Semiempirical Quantum Mechanical Methods for Noncovalent Interactions for Chemical and Biochemical Applications. Chem. Rev. 2016, 116, 53015337.

(41) Brandenburg, J. G.; Alessio, M.; Civalleri, B.; Peintinger, M. F.; Bredow, T.; Grimme, S. Geometrical Correction for the Inter- and Intramolecular Basis Set Superposition Error in Periodic Density Functional Theory Calculations. J. Phys. Chem. A 2013, 117, 92829292.

(42) Dovesi, R.; Orlando, R.; Erba, A.; Zicovich-Wilson, C. M.; Civalleri, B.; Casassa, S.; Maschio, L.; Ferrabone, M.; De La Pierre, M.; D'Arco, P.; et al. CRYSTAL14: A Program for the Ab Initio Investigation of Crystalline Solids. Int. J. Quantum Chem. 2014, 114, $1287-1317$.

(43) Orlando, R.; De La Pierre, M.; Zicovich-Wilson, C. M.; Erba, A.; Dovesi, R. On the full exploitation of symmetry in periodic (as well as molecular) self-consistent-field ab initio calculations. J. Chem. Phys. 2014, 141, 104108.
(44) Delle Piane, M.; Corno, M.; Orlando, R.; Dovesi, R.; Ugliengo, $\mathrm{P}$. Elucidating the fundamental forces in protein crystal formation: the case of crambin. Chem. Sci. 2016, 7, 1496-1507.

(45) Erba, A. On combining temperature and pressure effects on structural properties of crystals with standard ab initio techniques. $J$. Chem. Phys. 2014, 141, 124115.

(46) Erba, A.; Maul, J.; Itou, M.; Dovesi, R.; Sakurai, Y. Anharmonic Thermal Oscillations of the Electron Momentum Distribution in Lithium Fluoride. Phys. Rev. Lett. 2015, 115, 117402.

(47) Erba, A.; Shahrokhi, M.; Moradian, R.; Dovesi, R. On how differently the quasi-harmonic approximation works for two isostructural crystals: Thermal properties of periclase and lime. J. Chem. Phys. 2015, 142, 044114.

(48) Erba, A.; Maul, J.; De La Pierre, M.; Dovesi, R. Structural and elastic anisotropy of crystals at high pressures and temperatures from quantum mechanical methods: The case of $\mathrm{Mg} 2 \mathrm{SiO} 4$ forsterite. J. Chem. Phys. 2015, 142, 204502.

(49) Grimme, S.; Steinmetz, M. Effects of London Dispersion Correction in Density Functional Theory on the Structures of Organic Molecules in the Gas Phase. Phys. Chem. Chem. Phys. 2013, 15, $16031-16042$.

(50) Macrae, C. F.; Bruno, I. J.; Chisholm, J. A.; Edgington, P. R.; McCabe, P.; Pidcock, E.; Rodriguez-Monge, L.; Taylor, R.; van de Streek, J.; Wood, P. A. Mercury CSD 2.0 - new features for the visualization and investigation of crystal structures. J. Appl. Crystallogr. 2008, 41, 466-470.

(51) Perdew, J. P.; Burke, K.; Ernzerhof, M. Generalized Gradient Approximation Made Simple. Phys. Rev. Lett. 1996, 77, 3865-3568; erratum. Phys. Rev. Lett. 1997, 78, 1396.

(52) Grimme, S.; Antony, J.; Ehrlich, S.; Krieg, H. A consistent and accurate $a b$ initio parametrization of density functional dispersion correction (DFT-D) for the 94 elements H-Pu. J. Chem. Phys. 2010, 132, 154104.

(53) Schäfer, A.; Horn, H.; Ahlrichs, R. Fully optimized contracted Gaussian basis sets for atoms Li to Kr. J. Chem. Phys. 1992, 97, 25712577.

(54) Rossi, M.; Gasparotto, P.; Ceriotti, M. Anharmonic and Quantum Fluctuations in Molecular Crystals: A First-Principles Study of the Stability of Paracetamol. Phys. Rev. Lett. 2016, 117, 115702 .

(55) Lejaeghere, K.; Vanduyfhuys, L.; Verstraelen, T.; Van Speybroeck, V.; Cottenier, S. Is the error on first-principles volume predictions absolute or relative? Comput. Mater. Sci. 2016, 117, 390396. 